http://jmscr.igmpublication.org/home/ ISSN (e)-2347-176x ISSN (p) 2455-0450 crossref DOI: https://dx.doi.org/10.18535/jmscr/v9i8.04

\title{
Effectiveness of Frozen Shoulder Treatment Using Hydraulic Distension by Injecting Large Volume of Normal Saline
}

\author{
Author \\ Prabhav Majagaiyan Pokhrel ${ }^{{ }^{*}}$ \\ ${ }^{1}$ Department of Orthopaedics, Patan Academy of Health Sciences, Lagankhel, Lalitpur, Nepal \\ *Corresponding Author \\ Prabhav Majagaiyan Pokhrel \\ Department of Orthopaedics, Patan Academy of Health Sciences, Lagankhel, Lalitpur, Nepal
}

\begin{abstract}
Introduction: Frozen shoulder is an insidious, painful condition that results in gradual restriction of movement in the shoulder. It is a clinical diagnosis frequently made for patients with shoulder pain and limited motion. Though self-limiting, various modalities of treatment like physiotherapy, hot fomentation, oral analgesics are recommended. These can include intra- articular injections, capsular distension, manipulation under anaesthesia, and surgery. However, manipulation after anaesthesia and surgery are limited by poor accessibility; thus, the intra-articular injection of drugs, such as corticosteroids, and capsular distension after intra-articular injection are more commonly used. But none of them are gold standard treatment. This study is being done to determine the effectiveness and safety of capsular distension as a modality of treatment in terms of its functional outcome.

Method: 61 patients complaining of pain and stiffness in one shoulder for at least 6 weeks with restricted movement in two or more planes and undergoing physiotherapy were studied from the Out Patient Department (OPD) of National Medical College \& Teaching Hospital. A Large volume (approx. 60-80 ml) of Normal saline along with $2.5 \mathrm{ml}$ of $1 \%$ lidocaine was injected intraarticularly and the outcome was recorded at different intervals up to 6 months. The Outcome was computed in SS Pro 16.

Result: The study documented $54.10 \%$ of the patient had pain in the right shoulder and $45.90 \%$ of them had a complaint on their left side with male predominance (55.74\%). We reported after the injection of saline abduction improved up to $56.57^{\circ}$ and adduction up to $44.26^{\circ}$.

Conclusion: Outcome of large volume Normal Saline injection in case of Frozen Shoulder under local anaesthesia was found to be effective. Further study with large sample is recommended. It can be performed in areas with limited resources.

Keywords: Abduction, Adduction, Frozen Shoulder, Injection, Normal Saline, Pain.
\end{abstract}

\section{Introduction}

Adhesive capsulitis (frozen shoulder) is an insidious, painful condition that results in gradual restriction of movement in the shoulder. It is a clinical diagnosis frequently made for patients with shoulder pain and limited motion.
The incidence of frozen shoulder in the general population is approximately $2 \%$, but several conditions are associated with an increased incidence, including female sex, age more than 49 years, diabetes mellitus (five times more), cervical disc disease, prolonged immobilization, 
hyperthyroidism, stroke or myocardial infarction, the presence of autoimmune diseases and trauma. Common age group is between 40 and 70 years. Approximately $70 \%$ of the patients are female ${ }^{(4)}$.

Frozen shoulders are a frequent reason for OPD visits in orthopaedics department. Though our own statistics is not available; this seems to be very common in day to day practice which involves economically productive age group. In developing countries, like Nepal, it affects the economy of the country. The various methods of treatment has been mentioned in the books and journals including simple non-steroidal antiinflammatory drugs along with physiotherapy, use of intra-articular steroid injection, manipulation under anaesthesia, and of course capsular distension with fluid (hydro- dilatation) under local anaesthesia with or without ultrasound guidance. But none of them are gold standard treatment. The purpose of this study is to find out the efficacy of single episode of normal saline injection in treatment of frozen shoulder which is an easy, simple procedure, can be carried out on an OPD basis and might prove to be effective, safe with lesser side effects and cost effective, in our context with limited facilities and expertise.

\section{Methods}

The study was conducted at the Department of Orthopaedics of National Medical College and Teaching Hospital from August, 2013 to August, 2014. A prospective interventional study was conducted on 61 OPD patients complaining of pain and stiffness in one shoulder for at least 6 weeks with restricted movement in two or more planes and undergoing physiotherapy. The sex and ethnicity of the patients were defined. Patients with bony or neurological disorders, polyarthritis, reflex sympathetic dystrophy, past surgical history or history of steroid injection in the affected joint and patients with co-morbid conditions like C.O.P.D or with uncontrolled diabetes were excluded from the study. Change in range of movement of the joint was recorded after injecting Normal Saline. The photographs were taken as a document for all types of examinations on each side. The data were also entered into SSpro -2016 and was analyzed.

\section{Result}

Our observation showed that $54.10 \%$ of the patient had pain in the right shoulder and $45.90 \%$ of them had a complaint on their left side. After injecting Normal Saline and recurrently examining the patient for 6 months we found outstanding results. We saw that abduction movement improved up to $56.57^{\circ}$ and adduction up to $44.26^{\circ}$.

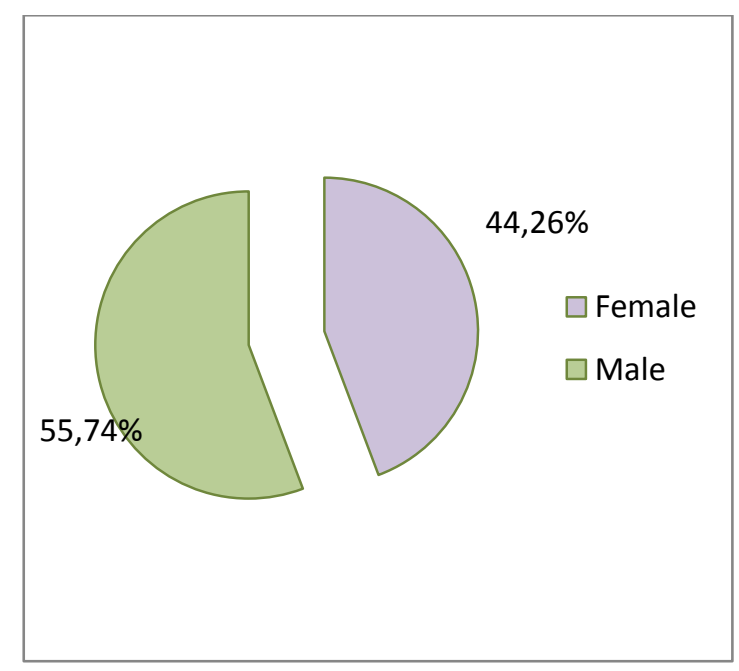

Figure 1: Pattern of sex distribution

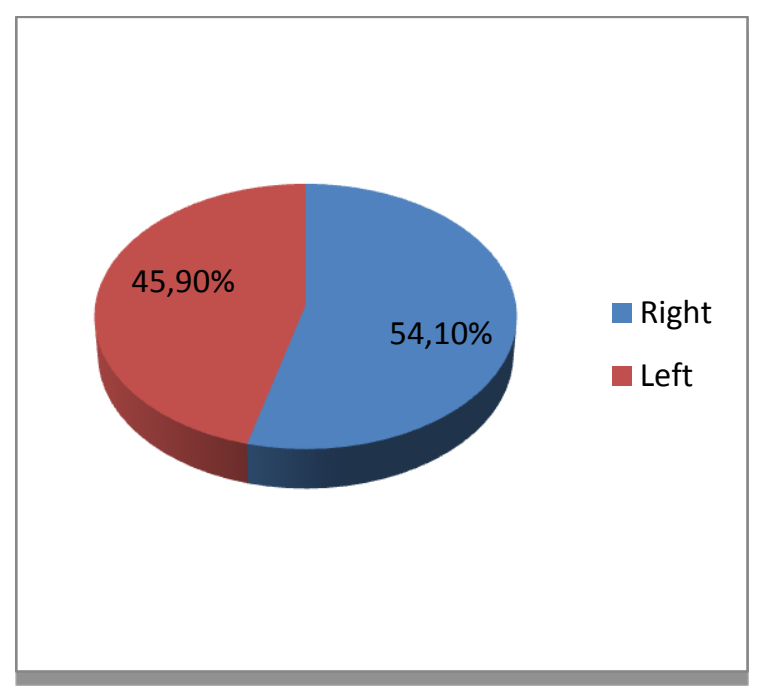

Figure 2: Pattern of side distribution 


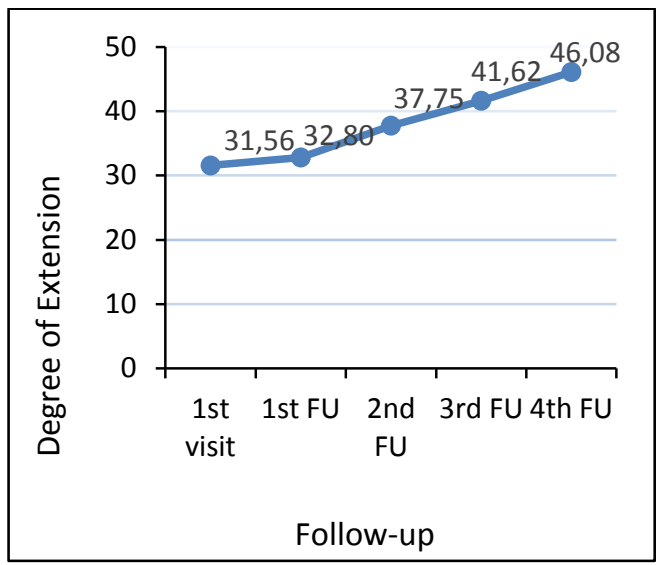

Fig 3: Showing degree of Extension

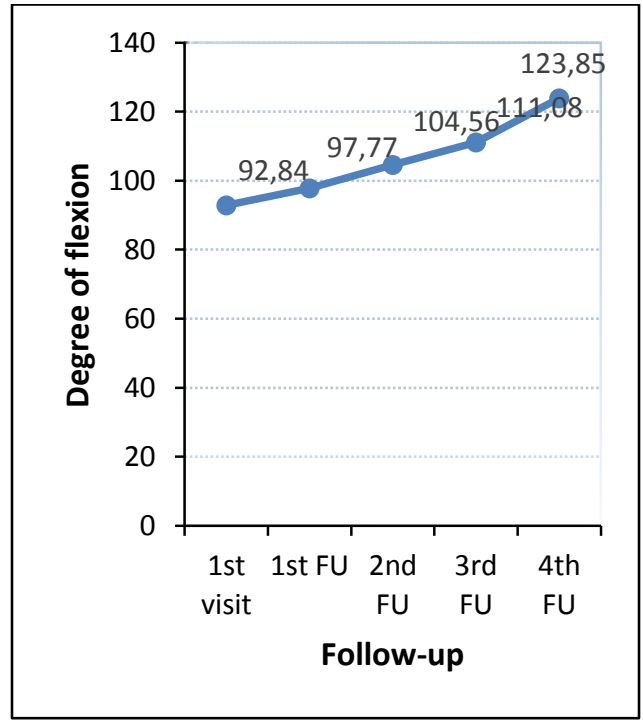

Fig 4: Showing degree of Flexion

Also on examining the extension and flexion movement after intralesional injection at different intervals during follow up we reported a significant increase in the degree of movement. We found extension increased up to $46.08^{\circ}$ and flexion up to $123.85^{\circ}$.

\section{Discussion}

Patients with shoulder pain and limited motion are usually diagnosed with frozen shoulder. Physiotherapy, hot fomentation, and oral analgesics are advised as therapeutic options, even if the condition is self-limiting. Intraarticular injections, capsular distension, manipulation under anesthesia, and surgery are some of the options. However, inadequate accessibility limits manipulation following anesthesia and surgery; as a result, intraarticular injections of medications like corticosteroids and capsular distension after intraarticular injection are more routinely employed, with mixed results.

Under local anesthesia and fluoroscopic monitoring, a needle is inserted into the joint capsule, and a mixture of long-acting anesthetic, cortisone, and saline is used to dilate the constricted joint capsule ${ }^{6}$.

In a study conducted by van Royen $\mathrm{BJ}^{\text {et }} \mathrm{al}^{7}$, the forward flexion of 24 frozen shoulder patients was found to be $94^{\circ}$ when compared to normal shoulder after hydraulic distension and manipulation. Our study reported an improvement between 92.83 to $123.85^{\circ}$. Our study showed beneficial effects in patients with frozen shoulders.

Kuptniratsaikul S et $\mathrm{al}^{8}$ studied 40 patients treating with capsular distention and MUA reported improved adduction by $31.4+/-7.2^{\circ}$. Adduction improvement in this study was just $11.18^{\circ}$. In patients with frozen shoulders, our research indicated that it was beneficial.

Callinan $\mathrm{N}$ et $\mathrm{al}^{9}$ reported increased abduction by $42^{\circ}$ in their study on the effectiveness of hydraulic distention combined with physiotherapy. Our study registered abduction increased by $56.57^{\circ}$. In patients with frozen shoulders, our research revealed positive results.

In research comparing capsular distension with intraarticular steroid injection alone, Gam AN et $\mathrm{al}^{10}$ observed no significant improvement in extension. Our study reported increased extension to be $14.52^{\circ}$. This difference might be because of the difference in the use of drugs during the different procedures.

\section{Conclusion}

A significant volume of normal saline injection was found to be safe and effective among various treatment techniques. The treatment modality should be tailored to the stage of the disease. Hydrodilatation is a cost-effective intervention in the management of Frozen Shoulder since it is safe, provides immediate and long-term pain relief, and improves range of motion. 


\section{Acknowledgements}

We would like to thank the ethical committee of National Medical College and Teaching Hospital, Birgunj for providing us the consent to conduct this study at this esteemed institute. We would also like to thank whole heartedly the administration and department without whom the study would not have been possible.

\section{Conflict of Interest: None}

Financial Disclosure: None

\section{References}

1. Tallia AF, Cardone DA. Diagnostic and therapeutic injection of the shoulder region. American family physician. 2003;67(6):1271-8.

2. Zuckerman JD, F C. The shoulder: a balance of mobility and stability. American Academy of Orthopaedic Surgery. 1993:p 19-109.

3. Canale ST, Beaty JH. Campbell's Operative Orthopaedics: Elsevier/Mosby; 2012.

4. Pearsall AW, Speer KP. Frozen shoulder syndrome: diagnostic and treatment strategies in the primary care setting. Medicine and science in sports and exercise. 1998;30(4 Suppl):S33-9.

5. Muller LP, Muller LA, Happ J, Kerschbaumer F. Frozen shoulder: a sympathetic dystrophy? Archives of orthopedic and trauma surgery. 2000;120(1-2):84-7.

6. Wybier M, Parlier-Cuau C, Baque MC, Champsaur P, Haddad A, Laredo JD. Distension Arthrography in Frozen Shoulder Syndrome. Seminars in musculoskeletal radiology. 1997;1(2):2516.

7. van Royen BJ, Pavlov PW. Treatment of frozen shoulder by distension and manipulation under local anesthesia.
International orthopedics. 1996;20(4):20710.

8. Kuptniratsaikul S, Kuptniratsaikul V, Tejapongvorachai T, Itiravivong P. A capsular dilatation facilitated shoulder manipulation for treating patients with frozen shoulder. Journal of the Medical Association of Thailand $=$ Chotmaihet thangphaet. 2002;85 Suppl 1:S163-9.

9. Callinan N, McPherson S, Cleaveland S, Voss DG, Rainville D, Tokar N. Effectiveness of hydroplasty and therapeutic exercise for treatment of frozen shoulder. Journal of hand therapy : official journal of the American Society of Hand Therapists. 2003;16(3):219-24.

10. Gam AN, Schydlowsky P, Rossel I, Remvig L, Jensen EM. Treatment of "frozen shoulder" with distension and glucocorticoid compared with glucocorticoid alone. A randomized controlled trial. Scandinavian journal of rheumatology. 1998;27(6):425-30. 